Volume 2, Issue 2, April-June 2017, Pages: 191, DOI: http://dx.doi.org/10.19082/ah191

\title{
FASA REGISTRY FOR SYSTOLIC HEART FAILURE (FARSH), FEASIBILITY STUDY
}

\author{
Ehsan Bahramali ${ }^{1}$, Azizallah Dehghan ${ }^{1}$, Mojtaba Farjam ${ }^{1}$ Habib Zakeri ${ }^{1}$, Alireza Askari ${ }^{1}$
}

Noncommunicable Diseased Research Center, Fasa University of Medical Sciences, Fasa, Iran

Correspondence:

Ehsan Bahramali MD. Tel: +989173118268, Fax:+987153316300, E-mail: ebahramali@gmail.com

\section{TYPE OF ARTICLE: CONFERENCE ABSTRACT}

\begin{abstract}
Background: Systolic heart failure (SHF), as a highly prevalent disease with serious costs to the health system, is becoming one of the major challenges for health policy makers. Every attempt to define treatment patterns and patient outcomes is welcome in that the definition of cost-effectiveness is of paramount importance for this chronic condition. A good surveillance system, which is missing in Iran, must thus consider both efficacy and safety measures. Fasa Registry for Systolic Heart failure (FaRSH) is the first hospital-based registry for SHF in Iran targeted to provide a meticulous description of patients' characteristics, to explore the management patterns of these patients, to discover the degree of adherence to the practice guidelines, and to investigate the determinants of poor in-hospital and later outcomes.

Methods: A diagnosis of SHF was made upon the accepted criteria by the attending cardiologists, and cases were further categorized to de-novo and chronic SHF. Two registrar nurses gathered data on demographics, place of residence and ethnicity, past medical history, risk factors, and the clinical course. Management patterns in the pre-hospital setting, during the hospital stay and at the discharge time were recorded. Routine laboratory results and cardiac biomarkers were registered.

Results: Pilot phase included the first 100 patients, the demographic, clinical and therapeutic constitutes of whom are presented in this article. Rate of re-hospitalization and other follow up measures are meticulously compiled and discussed.

Discussion: Obtained data on the characteristics of patients suffering from SHF revealed the major determinants of inadequate therapies and contributors of poor outcome. Completeness of data was guaranteed upon involvement of multiple checkpoints, and data quality was secured by means of automatic validation processes in addition to weakly physicians' roundups.

Conclusion: Execution of FaRSH in the form presented, is feasible and it will build up a comprehensive hospital-based registry for SHF in the region.

KEYWORDS: Disease registry, Systolic heart failure, Feasibility study
\end{abstract}

